\title{
First results from a high-resolution small animal PET insert for PET/MRI imaging
}

\author{
Andrew Goertzen ${ }^{1 *}$, Greg Stortz ${ }^{2}$, Jonathan Thiessen ${ }^{3}$, Daryl Bishop ${ }^{4}$, Muhammad Salman Khan ${ }^{1}$, Piotr Kozlowski ${ }^{2}$, \\ Fabrice Retiere ${ }^{4}$, Graham Schellenberg ${ }^{1}$, Vesna Sossi ${ }^{1}$, Christopher Thompson ${ }^{5}$
}

From PSMR 2015: 4th Conference on PET/MR and SPECT/MR

La Biodola, Isola d'Elba, Italy. 17-21 May 2015

${ }^{1}$ University of Manitoba, Canada
We have recently completed construction of a high resolution small animal PET insert designed for operation inside a Bruker 7T MRI. The PET insert is designed to achieve a $1 \mathrm{~mm}$ spatial resolution in the centre of its field of view (FOV) and fit within the $114 \mathrm{~mm}$ inner diameter of the Bruker BGA-12S gradient coil while accommodating the Bruker $35 \mathrm{~mm}$ volume RF coil (outer diameter $60 \mathrm{~mm}$ ). The PET insert is a ring geometry with a single ring of 16 detectors. Each detector uses a dual layer offset (DLO) LYSO scintillator array (bottom/top layer: $22 \times 10 / 21 \times 9$ of $1.2 \times 1.2 \times 6 / 4 \mathrm{~mm}$ crystals, 409 crystals per block), with total axial extent of $28.3 \mathrm{~mm}$, readout by two SensL SPMArray4B SiPM arrays. The detector outputs are multiplexed to four signals using a custom readout board and digitized using the OpenPET data acquisition platform. Detector flood image quality is sufficient to resolve $>99 \%$ of the crystals in the system. The average energy resolution of the 6544 crystals is $11.94 \%+/-1.77 \%$ at $511 \mathrm{keV}$. MR compatibility testing of the complete PET system conducted with a 7T Bruker Avance III MRI showed that the operating PET insert had no effect on MRI image homogeneity and only a small effect on EPI signal to noise ratio (SNR) (-15\%). Initial PET data were collected using a Ge68 line source with the PET system on the benchtop. For this first acquisition, the OpenPET system was operating in oscilloscope mode, limiting the total singles event rate to $18 \mathrm{kcps}$. The sinogram and initial reconstructed images showed no obvious artefacts. We have recently implemented an OpenPET firmware upgrade that will support a singles rate of $280 \mathrm{kcps}$; this will allow us to acquire first simultaneous phantom and mouse PET/MR images.

Authors' details

'University of Manitoba, Canada. ${ }^{2}$ University of British Columbia, Canada. ${ }^{3}$ Lawson Health Research Institute, Canada.

${ }^{4}$ TRIUMF, Canada. ${ }^{5}$ Montreal Neurological Institute, Canada.

Published: 18 May 2015

doi:10.1186/2197-7364-2-S1-A54

Cite this article as: Goertzen et al:: First results from a high-resolution small animal PET insert for PET/MRI

imaging. EJNMMI Physics 2015 2(Suppl 1):A54.

\section{SpringerOpen ${ }^{\odot}$}

(C) 2015 Goertzen et al; licensee Springer. This is an Open Access article distributed under the terms of the Creative Commons Attribution License (http://creativecommons.org/licenses/by/4.0), which permits unrestricted use, distribution, and reproduction in any medium, provided the original work is properly cited. 